\title{
Efeitos do treinamento resistido na capacidade funcional de pacientes com DPOC hospitalizados: revisão sistemática
}

\author{
Effects of resistance training on the functional capacity of \\ hospitalized COPD patients: systematic review
}

\author{
Laiane Costa Couto' ${ }^{1}$ Thiago Araújo de Melo² \\ ${ }^{1}$ Autora para correspondência. Centro Universitário do Instituto Social da Bahia. Salvador, Bahia, Brasil. \\ ORCID: 0000-0001-7103-4134. laianeccouto@gmail.com \\ ¿Universidade Salvador, Hospital Aliança. Salvador, Bahia, Brasil. ORCID: 0000-0001-6132-5616. thiago.melo@unifacs.br
}

RESUMO | INTRODUÇÃO: A reabilitação pulmonar (RP) vem sido descrita como uma forma de tratamento para pacientes com doença pulmonar obstrutiva crônica (DPOC), tendo o treino resistido como seu principal componente. Apesar de estar clara na literatura a importância e reais benefícios adquiridos com programa de reabilitação, este muitas vezes só é iniciado após a alta hospitalar, adiando os potenciais efeitos e intensificando o comprometimento funcional do paciente. OBJETIVO: Revisar sistematicamente as publicações presentes na literatura relacionados aos efeitos do treino resistido sobre a capacidade funcional de pacientes com DPOC, considerando a viabilidade, segurança e características da prescrição do exercício durante o processo de reabilitação. MATERIAIS E MÉTODOS: Trata-se de um estudo de revisão sistemática (CRD42020158760) sobre os efeitos do treino resistido sobre a capacidade funcional de pacientes com DPOC. Foram selecionados artigos científicos no período de setembro de 2018 a fevereiro de 2019 que continham os seguintes descritores: "DPOC", "treinamento de resistência", "fraqueza muscular", "hospitalização" e "hospital". RESULTADOS: Foram incluídos dois estudos nesta revisão sistemática atendendo aos critérios de elegibilidade. Ambos utilizaram como forma de avaliação da capacidade funcional o teste de caminhada de 6 minutos e obtiveram resultados favoráveis ao treinamento iniciado em ambiente hospitalar. CONCLUSÃO: $O$ treinamento resistido foi capaz de melhorar a capacidade funcional refletindo sobre a melhora na realização de atividades de vida diária e qualidade de vida. Demonstrou ser segura, de viável implementação e sem riscos para eventos adversos.

PALAVRAS-CHAVE: Doença pulmonar obstrutiva crônica. Treinamento de resistência. Fraqueza muscular. Hospitalização.
ABSTRACT | INTRODUCTION: Pulmonary rehabilitation (PR) has been described as a form of treatment for patients with chronic obstructive pulmonary disease, with resistance training as its main component. Although the importance and real benefits of a rehabilitation program are clear in the literature, it is often only started after hospital discharge, postponing the potential effects and intensifying the patient's functional impairment. OBJECTIVE: Systematically review publications in the literature regarding the effects of resistance training on the functional capacity of patients with COPD, considering the feasibility, safety and characteristics of exercise prescription during the rehabilitation process. MATERIALS AND METHODS: This is a systematic review study (CRD42020158760) of the effects of resistance training on the functional capacity of patients with COPD. Scientific articles were selected from september 2018 to february 2019 that contained the following descriptors: "COPD", "resistance training", "muscle weakness", "hospitalization" and "hospital". RESULTS: Two studies were included in this systematic review meeting the eligibility criteria. Both used the 6-minute walk test as a means of assessing functional capacity and had favorable effects on training initiated in a hospital setting. CONCLUSION: Resistance training was able to improve functional capacity reflecting on the improvement in the performance of activities of daily living and quality of life. It has been shown to be safe, feasible to implement and without risk for adverse events.

KEYWORDS: Chronic obstructive pulmonary disease. Resistance training. Muscle weakness. Hospitalization. 


\section{Introdução}

Pacientes hospitalizados com doença pulmonar obstrutiva crônica (DPOC) comumente apresentam elevada taxa de morbidade e mortalidade, além de redução significativa da qualidade de vida, número elevado de re-internações e aumento dos custos com recursos e serviços de saúde ${ }^{1}$. Quando em estado agudo, o tratamento desses pacientes fica restrito ao manejo da disfunção respiratória, e ao longo do processo da hospitalização por vezes subestimam-se os efeitos da doença sob a condição física ${ }^{2,3}$.

Além das alterações pulmonares, indivíduos com DPOC apresentam repercussões de caráter sistêmico, incluindo a disfunção generalizada dos músculos esqueléticos. A disfunção é representada por fraqueza muscular, especialmente de membros inferiores (MMII), redução de massa muscular, diminuição de resistência e presença de fadiga aos pequenos esforços ${ }^{4,5}$. Essas manifestações levam à redução do nível e da capacidade de realizar atividade física diária, limitação da tolerância ao exercício, contribuindo para perda de mobilidade, declínio funcional e redução da qualidade de vida ${ }^{6,7}$.

Programas de reabilitação pulmonar (PRP) têm sido empregados e indicados no tratamento de pacientes com doenças respiratórias crônicas, sintomáticos e que têm diminuição de atividades de vida diária ${ }^{8,9}$. De acordo com a American College of Chest Physicians e a American Association of Cardiovascular and Pulmonary Rehabilitation $^{10}$, a reabilitação pulmonar traz inúmeros benefícios ao paciente, incluindo melhora da tolerância ao exercício e qualidade de vida, além da redução da dispneia. O PRP pode ser realizado em indivíduos hospitalizados, em pacientes no âmbito ambulatorial ou domiciliar, sendo a segunda modalidade de tratamento a mais comumente realizada e a que possui maior descrição científica ${ }^{11}$.

Atualmente é crescente o número de evidências que abordam a importância do exercício, especialmente a modalidade de treino resistido como meio de favorecer o aumento da capacidade física, da massa e força muscular periférica, além da qualidade de vida nesse

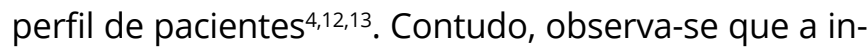
trodução desta modalidade de exercício como parte da reabilitação pulmonar (RP) é iniciada somente após a alta hospitalar, postergando os benefícios conseguidos com o tratamento e intensificando o comprometimento funcional do paciente ${ }^{6}$. Apesar do treinamento físico ter demonstrado ser um componente importante no cuidado ao paciente hospitalizado, poucos são os estudos que abordam seus efeitos, especialmente no DPOC, durante a fase de internação.

O objetivo deste estudo foi revisar sistematicamente as publicações presentes na literatura a respeito dos efeitos do treino resistido sobre a capacidade funcional de pacientes com DPOC.

\section{Materiais e método}

Esta revisão sistemática foi realizada de acordo com os critérios do PRISMA (Preferred Reporting Items for Systematic Reviews and Meta-Analyses) por dois investigadores. O protocolo de estudo foi registrado no banco internacional de registro de revisões sistemáticas (Prospero CRD42020158760).

\section{Estratégia de Busca}

O presente artigo caracteriza-se por ser uma revisão sistemática, na qual foram avaliados estudos nas bases de dados eletrônicas: Scientific Eletronic Library Online (SciELO), National Library of Medicine (Medline), Literatura Latino-Americana e do Caribe em Ciências da Saúde (Lilacs), Pubmed e Physiotherapy Evidence Database (PEDro), no período de setembro de 2018 a fevereiro de 2019, sem restrição para ano de publicação. Para cada base de dados foram utilizadas combinações com o operador booleano "AND", dos seguintes descritores: "DPOC", "treinamento de resistência", "fraqueza muscular", "hospitalização" e "hospital", com os correlatos em inglês: "COPD", "resistance training", "muscle weakness" e "hospitalization". A estratégia completa de pesquisa para a base de dados Pubmed consta no Quadro 1. 


\begin{tabular}{|c|}
\hline "COPD" AND "resistance training" \\
\hline "COPD" AND " muscle weakness" \\
\hline “COPD” AND “resistance training" AND “hospitalization” \\
\hline “COPD” AND “muscle weakness” AND “hospitalization” \\
\hline "DPOC" AND "treinamento de resistência" \\
\hline "DPOC" AND "fraqueza muscular" \\
\hline “DPOC" AND "treinamento de resistência" AND "hospitalização" \\
\hline “DPOC" AND "fraqueza muscular” AND “hospitalização” \\
\hline "DPOC" AND "hospital" \\
\hline "DPOC" AND "treinamento de resistência" AND “hospital” \\
\hline
\end{tabular}

\section{Critérios de Seleção}

A pesquisa foi limitada aos estudos realizados em idosos com DPOC em internação hospitalar. Foram incluídos ensaios clínicos randomizados escritos na língua portuguesa ou inglesa, envolvendo o treino resistido como componente de tratamento e seus efeitos sobre a capacidade funcional nesse perfil de pacientes. Foram excluídos estudos envolvendo outros componentes do programa de RP como o treinamento muscular respiratório ou treino aeróbico, programa de RP ambulatoriais e/ou domiciliares, os que não abordaram a capacidade funcional como desfecho.

\section{Avaliação da Qualidade Metodológica}

Os estudos encontrados foram sistematicamente analisados através de um instrumento específico de avaliação da qualidade metodológica. A escala PEDRo foi elaborada baseando-se na escala Delphi desenvolvida por Verhagen e colaboradores, sendo composta por 11 critérios. A pontuação final é dada pela soma do número desses critérios, sendo que o primeiro item não entra, podendo variar de 0 a 10 pontos. Quanto mais alta a pontuação, melhor é a qualidade do estudo ${ }^{14}$.

\section{Extração dos Dados}

A seleção dos artigos foi baseada na verificação e coerência dos títulos de cada estudo, seguidos de leitura dos resumos disponíveis. Aqueles elegíveis, foram posteriormente submetidos a leitura completa e avaliados quanto aos critérios de inclusão pré-estabelecidos. Ao final, um resumo crítico foi elaborado com síntese de informações disponibilizadas pelos artigos incluídos na revisão. 


\section{Resultados}

Na busca inicial realizada nas bases de dados foram identificados um total de 1.071 artigos. Destes, 868 foram excluídos pela leitura do título, em seguida, 203 artigos foram avaliados para leitura dos resumos, porém 36 foram considerados não relacionados ao tema. 152 artigos foram excluídos por serem repetidos. Após leitura completa dos artigos, 10 estudos foram excluídos, pois tratavam-se de reabilitação ambulatorial, 2 por abordarem o treino resistido associado a outro componente de exercício e 1 por não apresentar capacidade funcional como desfecho. Por fim, 2 estudos foram incluídos para esta revisão sistemática atendendo aos critérios de elegibilidade propostos. O fluxograma da estratégia de pesquisa é apresentado na figura 1.

Figura 1. Busca e seleção de estudos para inclusão na revisão sistemática de acordo com a metodologia PRISMA

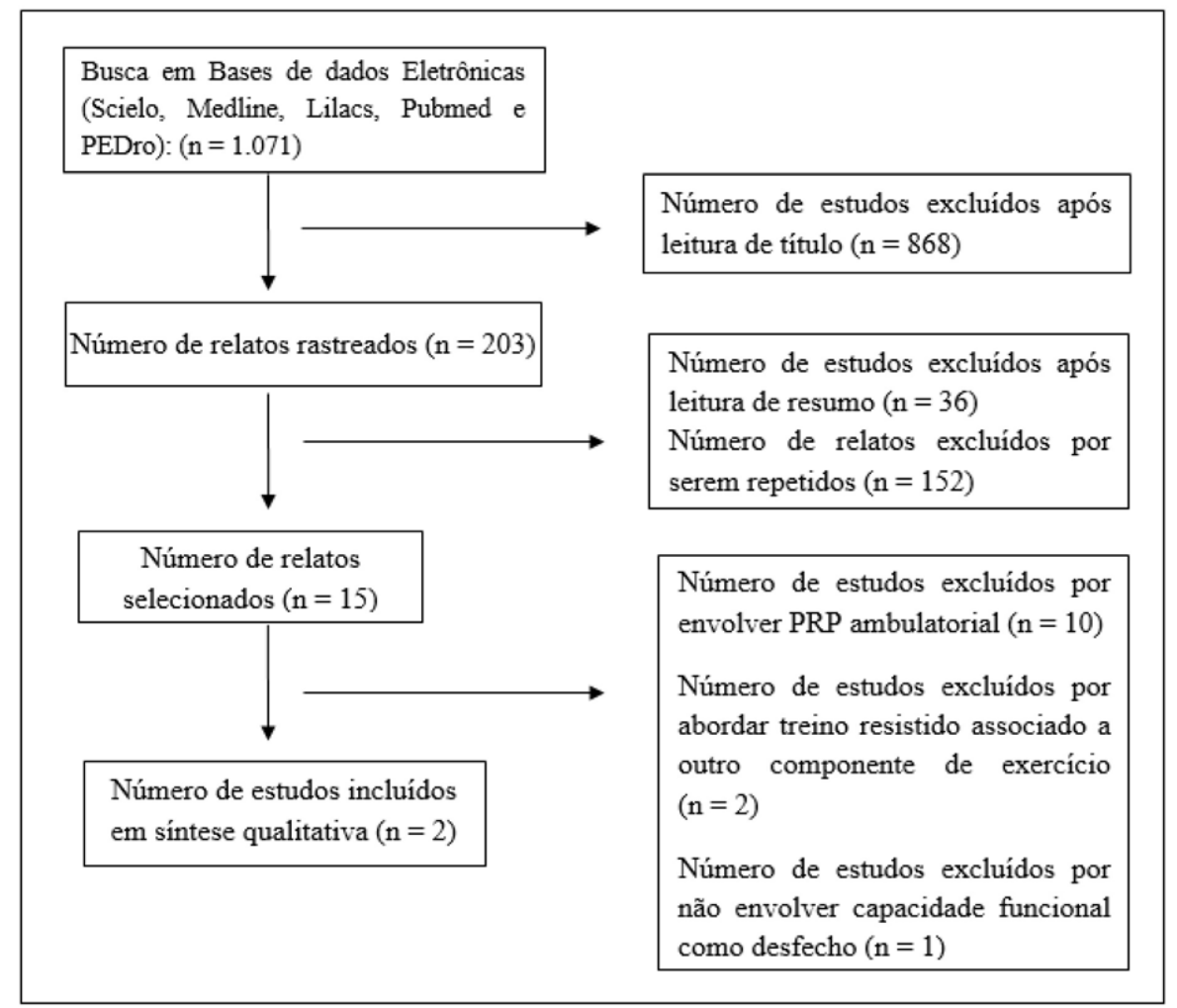

Em relação à qualidade metodológica dos artigos incluídos os dois estudos em análise foram considerados de alta qualidade, uma vez que atingiram pontuação superior a 5 na Escala PEDro. Os critérios avaliados pela escala e os escores obtidos por cada um dos estudos são apresentados de forma detalhada na Tabela 1. 
Tabela 1. Qualidade metodológica dos estudos pela Escala PEDro

\begin{tabular}{|c|c|c|c|c|c|c|c|c|c|c|c|c|}
\hline AUTOR & 1 & 2 & 3 & 4 & 5 & 6 & 7 & 8 & 9 & 10 & 11 & TOTAL \\
\hline Borges; Carvalho. 2014 & & $x$ & $x$ & $x$ & & & $x$ & & & $x$ & $x$ & 6 \\
\hline Troosters et al., $2010^{16}$ & $x$ & $x$ & $x$ & $x$ & & & & $x$ & & $x$ & $x$ & 6 \\
\hline \multicolumn{13}{|c|}{$\begin{array}{l}\text { 1) especificação dos critérios de inclusão (item não pontuado); 2) alocação aleatória; 3) sigilo na alocação; 4) similaridade } \\
\text { dos grupos na fase inicial ou basal; 5) mascaramento dos sujeitos; 6) mascaramento do terapeuta; 7) mascaramento do } \\
\text { avaliador; 8) medida de pelo menos um desfecho primário em } 85 \% \text { dos sujeitos alocados; 9) análise da intenção de tratar; } \\
\text { 10) comparação entre grupos de pelo menos um desfecho primário e 11) relato de medidas de variabilidade e estimativa } \\
\text { dos parâmetros de pelo menos uma variável primária. }\end{array}$} \\
\hline
\end{tabular}

Os artigos incluídos nesta revisão sistemática tiveram ano de publicação de 2010 e 2014 com casuísticas compostas por 36 e 29 sujeitos respectivamente, e idade média variando de 64 a 69 anos entre os participantes dos estudos. A tabela 2 apresenta a caracterização da amostra, perfil clínico dos participantes, protocolo de tratamento, frequência e forma de tratamento do treino resistido e desfechos de cada estudo incluído. Em ambos estudos o instrumento de avaliação para a variável capacidade de exercício foi o teste de caminhada de 6 minutos (TC6).

Tabela 2. Caracterização da amostra, metodologia, instrumento de avaliação da variável e desfechos dos estudos

\begin{tabular}{|c|c|c|c|c|c|c|c|}
\hline AUTOR & AMOSTRA & PERFIL CLÍNICO & $\begin{array}{l}\text { PROTOCOLO DE } \\
\text { TRATAMENTO }\end{array}$ & FREQUÊNCIA & $\begin{array}{l}\text { FORMA DE } \\
\text { REALIZAÇÃOO }\end{array}$ & $\begin{array}{l}\text { VARIÁVEL/ } \\
\text { INSTRUMENTO DE } \\
\text { AVALIAÇÃO }\end{array}$ & DESFECHOS \\
\hline $\begin{array}{l}\text { Borges e } \\
\text { Carvalho. } \\
2014^{15}\end{array}$ & $\begin{array}{l}29 \text { (idade média: } \\
\text { GC } 67.8 \pm 9.0, \text { GT } \\
64.1 \pm 12.5)\end{array}$ & $\begin{array}{l}\text { Pacientes com } \\
\text { DPOC } \\
\text { hospitalizados } \\
\text { devido } \\
\text { exacerbação da } \\
\text { doença, admitidos } \\
\text { na enfermaria. }\end{array}$ & $\begin{array}{l}\text { GC: Cuidados } \\
\text { habituais ( } 14 \text { sujeitos) } \\
\text { GT: Cuidados } \\
\text { habituais + programa } \\
\text { de treinamento de } \\
\text { resistência de MMSS } \\
\text { e MMII ( } 15 \text { sujeitos). }\end{array}$ & $\begin{array}{l}\text { Iniciado a partir do } \\
3^{\circ} \text { dia de } \\
\text { internamento com } \\
\text { sessões realizadas } \\
\text { todas as manhãs. } \\
\text { Pacientes } \\
\text { completaram o } \\
\text { mínimo de } 3 \\
\text { sessões durante o } \\
\text { estudo. }\end{array}$ & $\begin{array}{c}\text { Exercício de MMSS - } \\
\text { Flexão e abdução de } \\
\text { ombro, extensão de } \\
\text { cotovelo. Exercício de } \\
\text { MMII - flexão e } \\
\text { extensão de joelho e } \\
\text { flexão de quadril. } \\
\text { Realizados na } \\
\text { posição sentada, } \\
\text { utilizando pesos } \\
\text { livres, } 2 \text { x de } 8 \\
\text { repetições e carga } \\
\text { inicial de } 80 \% \text { de } \\
1 \text { RM. }\end{array}$ & $\begin{array}{l}\text { Capacidade de } \\
\text { exercício / TC6 }\end{array}$ & $\begin{array}{c}\text { - Treino seguro e de viável } \\
\text { implementação. - Não foi } \\
\text { observado aumento da } \\
\text { inflamação sistêmica ou } \\
\text { tempo de internação } \\
\text { hospitalar. } \\
\text { - Houve melhora da FM de } \\
\text { MMSS e MMII. } \\
\text { - Não houve efeitos } \\
\text { significativos sobre a QV } \\
\text { dos participantes. } \\
\text { - Apresentou melhora da } \\
\text { distância percorrida no TC6 } \\
\text { com maior aumento no GT, } \\
\text { sendo observada diferença } \\
\text { estatisticamente } \\
\text { significante intergrupo. }\end{array}$ \\
\hline $\begin{array}{l}\text { Troosters et } \\
\text { al., 2010 }\end{array}$ & $\begin{array}{c}36 \text { (idade média: } \\
\text { GC } 69 \pm 7, \text { GT } 67 \pm \\
\text { 8). }\end{array}$ & $\begin{array}{l}\text { Pacientes com } \\
\text { DPOC internados } \\
\text { com exacerbação } \\
\text { da doença, } \\
\text { admitidos na } \\
\text { enfermaria. }\end{array}$ & $\begin{array}{c}\text { GC: cuidados } \\
\text { habituais (19 sujeitos) } \\
\text { GT: cuidados } \\
\text { habituais + treino } \\
\text { diário de resistência } \\
\text { do quadriceps (17 } \\
\text { sujeitos). }\end{array}$ & 7 dias. & $\begin{array}{c}\text { Exercício utilizando } \\
\text { cadeira extensora de } \\
\text { joelhos realizado } 3 x \\
\text { de } 8 \text { repetições com } \\
\text { carga inicial de } 70 \% \\
\text { de } 1 \text { RM. }\end{array}$ & $\begin{array}{l}\text { Capacidade funcional / } \\
\text { TC6 }\end{array}$ & $\begin{array}{c}\text { - Treino seguro, viável e } \\
\text { eficaz. } \\
\text { - Sem impacto nos } \\
\text { marcadores de inflamação } \\
\text { sistêmica, no tempo de } \\
\text { internação hospitalar ou } \\
\text { taxa de readmissão. } \\
\text { - Observado aumento } \\
\text { significante da FM do } \\
\text { quadriceps sendo maior no } \\
\text { GT. } \\
\text { - Houve melhora na } \\
\text { distância percorrida no TC6 } \\
\text { no GT, porém sem } \\
\text { alteração no GC. Não } \\
\text { houve diferença } \\
\text { significante intergrupos. }\end{array}$ \\
\hline
\end{tabular}




\section{Discussão}

Hospitalizações por doenças agudas ou crônicas impõem aos pacientes internados certo grau de imobilidade, e o declínio funcional pode ser estabelecido de forma rápida, principalmente nos idosos. As atividades de vida diária (AVDs) e atividades instrumentais de vida diária (AIVs) podem ficar extremamente prejudicadas após período de internação, gerando nesses pacientes dificuldade para realiza-las de maneira autônoma ${ }^{17}$. Indivíduos com DPOC apresentam manifestações sistêmicas importantes, dentre elas, as alterações nutricionais e disfunção musculoesquelética, que resultam na diminuição da capacidade funcional. Exacerbações da doença com posterior período de internação hospitalar, torna-se motivo para intensificação do declínio funcional ${ }^{18,19}$.

Apesar de ser amplamente descrito na literatura a importância do PRP no tratamento de pacientes com DPOC, poucos são os estudos que abordam a sua realização no âmbito hospitalar, especialmente o componente de treinamento resistido. Após análise foi verificado que apenas dois estudos abordaram os efeitos isolados do treino sobre a capacidade funcional nesse perfil de pacientes.

O treinamento físico integra uma etapa do PRP. É composto pelo treino resistido de membros superiores, com utilização de pesos, faixas elásticas ou bastões, e de membros inferiores através dos exercícios de levantamento de pesos. A carga de treinamento comumente utilizada é baseada em um teste incremental inicial, adotando-se 50\% da carga máxima obtida ${ }^{11}$. Nos artigos analisados houve divergência quanto a carga de treinamento utilizada, ao volume de exercício e frequência de treinamento. $\mathrm{O}$ estudo de Borges e Carvalho15 adotou a carga correspondente a $80 \%$ de $1 \mathrm{RM}$, volume de exercício com 2 séries de 8 repetições e frequência mínima de 3 sessões durante todo o estudo. Já Troosters et al. ${ }^{16}$ adotou correspondente a $70 \%$ de $1 \mathrm{RM}$, volume de exercício com 3 séries de 8 repetições e frequência de treino diária por 7 dias. Em ambos os estudos o ajuste de carga foi realizado com base nos sintomas de dispnéia e fadiga dos participantes.
A capacidade funcional reflete a habilidade física que os indivíduos possuem para executar tarefas cotidianas. Ao contrário, a incapacidade funcional representa a dificuldade ou necessidade de ajuda externa para executar suas atividades básicas e instrumentais de vida diária, bem como aquelas relacionadas à mobilidade ${ }^{20,21}$. No DPOC o comprometimento funcional acarreta em aumento nos episódios de exacerbação da doença, internação hospitalar e mortalidade. Dessa forma, a capacidade funcional se torna imprescindível na avaliação desses pacientes e consequente marcador no planejamento adequado do PRP22.

Há diversas maneiras de se avaliar a capacidade funcional, dentre elas os questionários e testes funcionais. A Lawton e Brody e o Índice de Katz são utilizadas para avaliar atividades instrumentais e básicas de vida diária, respectivamente ${ }^{20}$. Dentre os testes mais utilizados na prática clínica têm-se o teste de caminhada de 6 minutos (TC6), o qual é amplamente realizado devido a sua simples execução, o seu baixo custo e validade clínica. A variável de desfecho do teste é a distância percorrida, fazendo referência de que quanto maior, melhor é a capacidade funcional do indivíduo ${ }^{22,23}$.

Ambos os artigos incluídos no estudo utilizaram o TC6 como método de avaliação e comparativo da capacidade funcional pré e pós tratamento. Um aumento de 54 m na distância percorrida no TC6 tradicionalmente demonstra ser clinicamente significativo ${ }^{24,25}$. No estudo de Borges e Carvalho15, durante a internação, os pacientes do grupo de treinamento exibiram no teste um aumento de $160( \pm 61) \mathrm{m}$, comparado ao grupo controle, que apresentou um aumento médio de $11( \pm 83) \mathrm{m}$. A melhora clinicamente significante observada no TC6 do grupo de treinamento se manteve nos pacientes até 1 mês pós alta hospitalar. No estudo de Troosters et al. ${ }^{16}$, no entanto, a distância no TC6 melhorou no grupo de treinamento após a alta (61-14 $\mathrm{m} ; \mathrm{P}=0.002)$, enquanto permaneceu inalterada no grupo controle $(62-41 \mathrm{~m} ; \mathrm{p}=0.59)$. Apesar de existir diferença entre os grupos, esta não foi significativa $(p=0.23)$. 
A maioria dos estudos disponíveis na literatura traz abordagem ao paciente e seu encaminhamento para reabilitação quando eles são considerados portadores de DPOC estável. No entanto, alguns estudos vêm demonstrando que o programa de exercícios pode ser considerado seguro e de viável implementação durante exacerbação aguda da doença. Tanto no artigo de Borges e Carvalho ${ }^{15}$ quanto no de Troosters et al. ${ }^{16}$, efeitos positivos ao grupo de treinamento foram observados. Não houve aumento dos níveis de marcadores inflamatórios, efeitos adversos durante tratamento ou aumento do tempo de internação. Esses resultados foram semelhantes a um estudo que avaliou um programa de exercícios de resistência associado a um treino aeróbico afim de determinar sua segurança e viabilidade ${ }^{26}$. Foi visto que o programa nos grupos de treinamento foi benéfico na melhora da tolerância ao exercício observado através do aumento da distância no teste de caminhada de 3 minutos após tratamento, além de não ter apresentado aumento do tempo de internação. Esses resultados indicam que o exercício durante período de exacerbação aguda não afetou negativamente a recuperação de pacientes com DPOC.

Como limitações, Borges e Carvalho ${ }^{15}$ consideraram a amostra de pacientes incluídos no estudo pequena, bem como uma porcentagem considerada grande na perda no seguimento do protocolo de tratamento. Além disso, a duração prolongada de algumas sessões do treino de resistência, em torno de 90 minutos, dificulta a sua implementação na prática clínica. No entanto, o estudo revela que trabalhar menos grupos musculares reduziria o tempo de atendimento tornando o protocolo mais reprodutível. Troosters et al. ${ }^{16}$ consideraram que estudos maiores são necessários para investigar o impacto do treino resistido nos resultados a longo prazo. Esses fatores de limitação demonstram a necessidade da realização de novos estudos para que haja melhor reprodutibilidade dos dados.

\section{Conclusão}

Baseado nessa revisão sistemática, a reabilitação pulmonar tendo o componente de treino resistido como foco, mostrou resultados benéficos quando iniciado em âmbito hospitalar em pacientes com DPOC na fase de exacerbação aguda da doença. O treinamento foi capaz de melhorar a capacidade funcional dos pacientes refletida através do aumento na distância do TC6 minutos. Além disso, o exercício prescrito nesta modalidade demonstrou-se seguro, de viável implementação em virtude da baixa frequência e sem riscos de eventos adversos observados durante o tratamento.

Devido à alta incidência da doença e o comprometimento funcional refratário a mesma, faz-se necessário o desenvolvimento de novos estudos, sobretudo ensaios clínicos randomizados com amostras mais representativas no intuito de avaliar o impacto do exercício resistido na condição funcional desta população a curto e longo prazo. Adicionalmente, novos estudos facilitarão o estabelecimento de recomendações mais sólidas a respeito da configuração do treinamento físico neste cenário.

\section{Contribuições dos autores}

Couto LC se responsabilizou pela execução da estratégia de busca, compilação e análise das informações, avaliação de qualidade metodológica e escrita do artigo. Melo TA se responsabilizou pela concepção do estudo, revisão metodológica e avaliação da qualidade final do artigo.

\section{Conflitos de interesses}

Nenhum conflito financeiro, legal ou político envolvendo terceiros (governo, empresas e fundações privadas, etc.) foi declarado para nenhum aspecto do trabalho submetido (incluindo mas não limitando-se a subvenções e financiamentos, participação em conselho consultivo, desenho de estudo, preparação de manuscrito, análise estatística, etc.). 


\section{Referências}

1. Rodrigues F. Importância de fatores extrapulmonares depressão, fraqueza muscular, qualidade de vida - na evolução da DPOC. Revista Portuguesa de Pneumologia. 2010;16(5): 709-715.

2. Silva APP, Maynard K, Cruz MR. Efeitos da fisioterapia motora em pacientes críticos: revisão de literatura. Revista Brasileira de Terapia Intensiva. 2010; 22(1): 85-91. doi: 10.1590/S0103$\underline{507 \times 2010000100014}$

3. Greening NJ, Williams JEA, Hussain SF, Harvey-Dunstan TC, Bankart MJ, Chaplin EJ, et al. An early rehabilitation intervention to enhance recovery during hospital admission for an exacerbation of chronic respiratory disease: randomised controlled trial. Brazilian Journal of Microbiology (Bjm). 2014;349:g4315. doi: 10.1136/bmj.g4315

4. Silva EGD, Dourado VZ. Treinamento de força para pacientes com doença pulmonar obstrutiva crônica. Rev Bras Med Esporte. 2008;14(3):231-238. doi: 10.1590/S1517-86922008000300014

5. Nellessen AG, Donária L, Hernandes NA, Pitta F. Análise de três diferentes fórmulas de predição de força muscular do quadríceps femoral em pacientes com DPOC. Jornal Brasileiro de Pneumologia. 2015; 41(4): 305-312. doi: 10.1590/S180637132015000004515

6. Trevisan ME, Porto AS, Pinheiro TM. Influência do treinamento da musculatura respiratória e de membros inferiores no desempenho funcional de indivíduos com DPOC. Fisioterapia e Pesquisa. 2010; 17(3): 209-213. doi: 10.1590/S180929502010000300004

7. Chiavegato LD. Avaliação da força do quadríceps como ferramenta para se estabelecer a conduta e o prognóstico em pacientes com DPOC. J Bras Pneumol. 2015;41(4):297-298. doi: 10.1590/S1806-37132015000400002

8. Jenkins S, Hill K, Cecins NM. State of the art: how to set up a pulmonary rehabilitation program. Respirology. 2010;15(8):11571173. doi: 10.1111/j.1440-1843.2010.01849.x

9. Zanchet RC, Viegas CAA, Lima T. A eficácia da reabilitação pulmonar na capacidade de exercício, força da musculatura inspiratória e qualidade de vida de portadores de doença pulmonar obstrutiva crônica. J Bras Pneumol. 2005;31(2):118-124. doi: 10.1590/S1806-37132005000200006
10. Ries AL, Bauldoff GS, Carlin BW, Casaburi R, Emery CF, Mahler DA et al. Pulmonary Rehabilitation: Joint ACCP/AACVPR EvidenceBased Clinical Practice Guidelines. Chest. 2007; 131(5):4S-42S. doi: 10.1378/chest.06-2418

11. Ribeiro BV. Reabilitação pulmonar: da teoria à prática. Pulmão RJ. 2015;24(3):54-58.

12. Silva BSA, Gobbo LA, Freire APCF, Trevisan IB, Silva IG, Ramos EMC. Effects of a resistance training with elastic tubing in strength, quality of life and dypsnea in patients with chronic obstructive pulmonary disease. J Phys Educ. 2016;27(1): 2722. doi: 10.4025/ jphyseduc.v27i1.2722

13. Dourado VZ, Godoy I. Recondicionamento muscular na DPOC: principais intervenções e novas tendências. Rev Bras Med Esporte. 2004;10(4):331-334. doi: 10.1590/S151786922004000400010

14. Shiwa SR, Costa LOP, Moser ADL, Aguiar IC, Oliveira LVF. PEDro: a base de dados de evidências em fisioterapia. Fisioterapia e Movimento. 2011;24(3):523-33. doi: 10.1590/S010351502011000300017

15. Borges RC, Carvalho CR. Impact of resistance training in chronic obstructive pulmonary disease patients during periods of acute exacerbation. Archives of physical medicine and rehabilitation. 2014; 95(9):1638-1645. doi: 10.1016/j. apmr.2014.05.007

16. Troosters T, Probst VS, Crul T, Pitta F, Gayan-Ramirez G, Decramer $\mathrm{M}$ et al. Resistance training prevents deterioration in quadriceps muscle function during acute exacerbations of chronic obstructive pulmonary disease. Am J Respir Crit Care Med. 2010;181(10):1072-7. doi: 10.1164/rccm.200908-12030C

17. Menezes C, Oliveira VRC, De Menezes RL. Repercussões da hospitalização na capacidade funcional de idosos. Revista Movimenta. 2010;3(2):76-84.

18. Karloh M, Palú M, Mayer AF. Métodos de avaliação da capacidade funcional em pacientes com DPOC. ConScientiae Saúde. 2014;13(4):633-649. doi: 10.5585/ConsSaude.v13n4.4830

19. Silva KR, Marrara KT, Marino DM, Di Lorenzo VAP, Jamami M. Fraqueza muscular esquelética e intolerância ao exercício em pacientes com doença pulmonar obstrutiva crônica. Rev Bras Fisioter. 2008;12(3):169-75. doi: 10.1590/S141335552008000300003 
20. Barbosa BR, De Almeida JM, Barbosa MR, Rossi-Barbosa LAR. Avaliação da capacidade funcional dos idosos e fatores associados à incapacidade. Ciênc Saúde Coletiva. 2014; 19(8):3317-3325. doi: 10.1590/1413-81232014198.06322013

21. César CC, Mambrini JVM, Ferreira FR, Lima-Costa MF. Capacidade funcional de idosos: análise das questões de mobilidade, atividades básicas e instrumentais da vida diária via Teoria de Resposta ao Item. Cadernos de Saúde Pública. 2015;31(5):931-945. doi: 10.1590/0102-311X00093214

22. Gulart AA, Santos K, Munari AB, Karloh M, Cani KC, Mayer AF. Relação entre a capacidade funcional e a percepção de limitação em atividades de vida diária de pacientes com DPOC. Fisioter Pesq. 2015;22(2):104-111. doi: 10.590/18092950/12836522022015

23. Carpes MF, Mayer AF, Simon KM, Jardim JR, Garrod R. Versão brasileira da escala London Chest Activity of Daily Living para uso em pacientes com doença pulmonar obstrutiva crônica. J Bras Pneumol. 2008;34(3):143-151. doi: 10.1590/S180637132008000300004

24. Redelmeier DA, Bayoumi AM, Goldstein RS, Guyatt GH. Interpreting small differences in functional status, the six minuts walk test is chronic lung disease patients. Am J Respir Crit Care Med. 1997;155(4):1278-82. doi: 10.1164/ajrccm.155.4.9105067

25. Berton DC, Silveira L, Costa CC, Souza RM, Winter CD, Teixeira PJZ. Effectiveness of pulmonary rehabilitation in exercise capacity and quality of life in chronic obstructive pulmonary disease patients with and without global fat-free mass depletion. Archives of physical medicine and rehabilitation. 2013;94(8):1607-14. doi: 10.1016/j.apmr.2013.02.005

26. Tang CY, Blackstock FC, Clarence M, Taylor NF. Early rehabilitation exercise program for inpatients during an acute exacerbation of chronic obstructive pulmonary disease: a randomized controlled trial. J Cardiopulm Rehabil Prev. 2012;32(3):163-169. doi: 10.1097/HCR.0b013e318252f0b2 